\title{
Anticancer efficacy of perillyl alcohol-bearing PLGA microparticles
}

\author{
This article was published in the following Dove Press journal: \\ International Journal of Nanomedicine \\ 4 January 2012 \\ Number of times this article has been viewed
}

\section{Mohammad Farazuddin' \\ Bhawna Sharma ${ }^{2}$ \\ Aijaz Ahmed Khan ${ }^{3}$ \\ Beenu Joshi ${ }^{2}$ \\ Mohammad Owais' \\ 'Interdisciplinary Biotechnology \\ Unit, Aligarh Muslim University, \\ Aligarh, Uttar Pradesh, India; \\ ${ }^{2}$ Immunology Division, NJIL and other Mycobacterial diseases, Agra-28200I \\ Uttar Pradesh, India; ${ }^{3}$ Department of Anatomy, JN Medical college, Aligarh Muslim University, Aligarh-202002, \\ Uttar Pradesh, India}

Correspondence: Mohammad Owais Interdisciplinary Biotechnology Unit, Aligarh Muslim University, Aligarh-202002, Uttar Pradesh, India $\mathrm{Tel}+9$ l 057| 2720388

Fax +9| 057| 2721776

Email owais_lakhnawi@yahoo.com
Abstract: In the present study, a novel poly-lactic glycolic acid (PLGA)-based microparticle formulation of perillyl alcohol $(\mathrm{POH})$ was prepared and characterized. Further, its efficacy was evaluated against di-methyl benzo anthracene-induced skin papilloma in Swiss albino mice. The characterization studies showed that POH-bearing PLGA microparticles were of the size $768 \pm 215 \mathrm{~nm}$ with a $\zeta$-potential value of $-7.56 \pm 0.88 \mathrm{mV}$. The entrapment efficiency of the active drug in particles was $42.4 \% \pm 3.5 \%$. POH-bearing PLGA microparticles were stable and released entrapped drug gradually over an extended time period. The in vitro efficacy of POH-bearing PLGA microparticles was evaluated by examining their differential cytotoxicity and assessing their ability to inhibit epidermoid carcinoma cell line (A253). The POH-based microparticles when administered to tumor-bearing animals caused greater tumor regression and increased survival rate $(\sim 80 \%)$ as compared with the group receiving free form of $\mathrm{POH}$ (survival rate $40 \%$ ). The superiority of POH-PLGA microparticles over free form of POH was further evident from their ability to modulate apoptosis-regulating factors.

Keywords: poly-lactic glycolic acid, epidermoid cancer cells, skin papilloma, anticancer efficacy

\section{Introduction}

Cytotoxicity and other related side effects are the most serious problems associated with the currently available anticancer drugs. Other limitations include widespread systemic distribution and rapid elimination of the administered anticancer drugs from the host body. A worldwide search therefore continues for anticancer drugs that are more potent, less toxic, and manifest minimum untoward effects to the host. Several plant derived compounds have been reported to possess strong anticancer properties and have been shown to delay, inhibit, or reverse cancerous growth in an effective manner. For example, perillyl alcohol $(\mathrm{POH})$, a plant-based compound, has been reported to possess strong anti-cytotoxic properties against several types of cancer including those of breast, pancreatic, and liver. ${ }^{1-3} \mathrm{POH}$ is a monoterpene and constituent of essential oils from a number of plants; namely, perilla (Perilla frutescens), lavendin, peppermint, ginger grass, savin, caraway, and celery seeds. ${ }^{4}$

Before translating the suitability of a novel compound like $\mathrm{POH}$ as a potential anticancer agent in the clinical setting, it is desirable to address some of the associated issues like that of its solubility, palatability, and sustained/controlled release in systemic circulation. This requires designing of a suitable drug-delivery system that can release the drug gradually over a long period of time and, in turn, facilitate its uptake by cancer cells and thereby helps in increasing the efficacy of the entrapped drug. Polymeric 
microparticles offer a promising technology in this regard. Encapsulation of drugs in the core of microparticles have been shown not only to protect them from the external environment enroute, but also help in increasing their plasma half-lives in systemic circulation thereby facilitating the attainment of optimum drug availability at the desired target. ${ }^{5-7}$

To develop promising and effective formulations of anticancer compounds, various polymeric matrices have been investigated and their efficacies worked out. ${ }^{5-10}$ Drugdelivery systems, such as nanoparticle-,${ }^{5}$ microemulsion- ${ }^{6}$ nanoemulsion- ${ }^{7}$ and liposome-based delivery systems, ${ }^{8-10}$ have been shown to enhance the efficacy of various compounds on systemic as well as topical applications. Among various drug-carrier systems, poly-lactic glycolic acid (PLGA) matrices have been reported to be nontoxic, biodegradable, and shown to release the entrapped drug gradually over a long duration. ${ }^{11}$

In the present study, POH-bearing PLGA microparticles were prepared and their efficacy against the skin epidermoid cancer cell line (A253) was evaluated. In addition, potential of POH-bearing PLGA microparticles was evaluated in treatment of di-methyl benzo anthracene (DMBA)-induced tumors in Swiss albino mice.

\section{Materials and methods}

All reagents used in the study were of highest purity available. PLGA, poly vinyl alcohol (PVA), and POH were purchased from Sigma Chemical Company (St Louis, MO). Dichloromethane (DCM) was of analytical grade of purity and procured locally. Anti-p53 mutant, anti-p53 wild-type (wt), anti-p21/waf1, anti-tubulin and anti- $\beta$-actin antibodies were purchased from BD Biosciences (San Diego, CA).

\section{Preparation of POH-loaded PLGA microparticles}

Microparticles used in the present study were prepared by oil-in-water-based emulsion solvent evaporation technique using the published protocol as standardized in our laboratory. ${ }^{12,13}$ Briefly, a known quantity of POH (30 mg) was dissolved in minimum volume of methanol, mixed with PLGA solution (190 mg PLGA dissolved in $1.0 \mathrm{~mL}$ DCM), and sonicated in a bath-type sonicator to form the primary emulsion. The primary emulsion was mixed with $100 \mathrm{~mL}$ of 10\% PVA (w/v) and homogenized using a Silverson L4RT homogenizer (Silverson Machines, East Longmeadow, MA). The resulting oil-in-water emulsion was stirred at $25^{\circ} \mathrm{C}$ for 18 hours to allow solvent evaporation and formation of $\mathrm{POH}-$ entrapped microparticles. The microparticles were centrifuged and thoroughly washed with phosphate buffered saline (PBS) (0.15 M NaCl containing $20 \mathrm{mM}$ sodium phosphate, $\mathrm{pH}$ 7.4) to remove surface adsorbed drug. The microformulation was lyophilized and finally stored at $4^{\circ} \mathrm{C}$ until further use.

\section{Entrapment efficiency of $\mathrm{POH}$ in microparticles}

Entrapment of $\mathrm{POH}$ in microparticles was assessed by dissolving an aliquot of the microparticles in $0.1 \mathrm{~N} \mathrm{NaOH}$ followed by analysis of $\mathrm{POH}$ content by high-performance liquid chromatography (HPLC) following the published procedure. ${ }^{14}$ Briefly, $10 \mathrm{mg}$ freeze-dried microparticles were dissolved in $1.0 \mathrm{~mL}$ of $0.1 \mathrm{~N} \mathrm{NaOH}$. The solution was vortexed for 10 minutes followed by centrifugation for 5 minutes at $9168 \times g$ at $25^{\circ} \mathrm{C}$. An aliquot $(100 \mu \mathrm{L})$ of supernatant was mixed with $900 \mu \mathrm{L}$ methanol. The suitable aliquots, of the resultant homogenate solution, were analyzed by reversed phase HPLC using a Symmetry ${ }^{\circledR}$ C-18 column $(3.9 \mathrm{~mm} \times 150 \mathrm{~mm})$. The solvent system used was isocratic methanol-water (72:28, v/v). Entrapment of $\mathrm{POH}$ was calculated with the help of calibration curve using the pure drug plotted at $220 \mathrm{~nm}$. The percentage entrapment efficiency (\% EE) was calculated with the following formula.

$\% \mathrm{EE}=($ amount of $\mathrm{POH}$ entrapped $)$

$/($ total amount of $\mathrm{POH}$ used in the beginning $) \times 100$.

\section{Scanning electron microscopy of the microparticles}

Scanning electron microscopy (SEM) was performed to characterize the size and surface morphology of POH-loaded microparticles using scanning electron microscope (Zeiss EVO 40; Carl Zeiss SMT AG, Oberkochen, Germany). The lyophilized preparation of $\mathrm{POH}$-loaded microparticles was suspended in $20 \mathrm{mM}$ PBS pH 7.4, and a drop of the formulation was mounted on clear glass stub, air dried, and coated with gold-palladium alloy using a sputter coater. An accelerating voltage of $20.00 \mathrm{kV}$ was used for imaging.

\section{Determination of $\zeta$-potential}

$\zeta$-potential of the PLGA microparticles was determined using DTS software (Malvern Instruments Ltd, Worcestershire, UK) based on M3-PALS technology. The formulation was lyophilized in a $2.0 \mathrm{~mL}$ microfuge tube, and the samples were reconstituted in $20 \mathrm{mM}$ phosphate buffer, $\mathrm{pH}$ 7.4. This dispersion was then rapidly dispensed to a electrophoresis cell to measure the electrophoretic mobility, and $\zeta$-potential 
values were calculated. The experiment was repeated three times, and the average $\zeta$-potential with standard deviation was calculated.

\section{In vitro release kinetics of active $\mathrm{POH}$ from PLGA microparticles}

To assess the release kinetics of POH from PLGA microparticles, multiple weighed aliquots of the microparticles were dispensed in separate microvials. To each vial, $1.0 \mathrm{~mL}$ of $20 \mathrm{mM}$ sterile PBS was added, followed by incubation at $37^{\circ} \mathrm{C}$. Aliquots $(100 \mu \mathrm{L})$ of supernatant were removed after centrifugation at $9168 \times g$ for 10 minutes and analyzed for the $\mathrm{POH}$ content.

\section{Toxicity tests for POH-bearing PLGA microformulation}

A new formulation of a given drug molecule has to be tested for any inherent toxicity before being examined for its efficacy. To settle this issue, toxicity of the in-house prepared formulation was tested both in vitro and in vivo. Preliminary acute drug toxicity was based on in vitro erythrocyte lysis test, wherein hemoglobin, released as a result of membrane leakage or disruption caused by exposure to low doses of the drug, is measured..$^{15}$ Briefly, fresh blood was obtained from a healthy rabbit and collected in anticoagulant solution (ethylenediaminetetraacetic acid), followed by centrifugation at $1000 \times g$ for 10 minutes at $4^{\circ} \mathrm{C}$. Buffy coat as well as plasma was discarded. The washed erythrocytes were diluted with isotonic buffer (20 mM PBS), and 50\% hematocrit was prepared. To study the extent of hemolysis, the suspension of red blood cells (RBCs) was incubated with $1.0 \mathrm{~mL}$ of free form as well as $\mathrm{POH}$ encapsulated in PLGA microparticles $(10 \mathrm{mg} / \mathrm{mL})$ at $37^{\circ} \mathrm{C}$ for 1 hour. Free $\mathrm{POH}$ was dissolved in $50 \mu \mathrm{L}$ of dimethyl sulfoxide (DMSO), and finally volume was made up to $1.0 \mathrm{~mL}$ with PBS (final 5\% DMSO). After 1 hour, the reaction mixture was centrifuged at $1500 \times g$, and supernatant was collected and analyzed by ultraviolet-visible spectroscopy $\left(\lambda_{\max }=576 \mathrm{~nm}\right)$ for released hemoglobin. The percentage hemolysis was determined by the following equation:

$$
\left.\left[\left(\mathrm{Abs}_{\mathrm{T}}-\mathrm{Abs}_{\mathrm{C}}\right) / \mathrm{Abs}_{100 \%}-\mathrm{Abs}_{\mathrm{C}}\right)\right] \times 100,
$$

where $\mathrm{Abs}_{\mathrm{T}}$ is the absorbance of the supernatant from samples incubated with the drugs, $\mathrm{Abs}_{\mathrm{C}}$ is the absorbance of the supernatant from controls (PBS), and $\mathrm{Abs}_{100 \%}$ is the absorbance of the supernatant of controls incubated in the presence of $1 \%$ Triton $^{\circledR} \mathrm{X}-100$, which causes complete lysis of RBCs (total hemolysis).
Hepatic and renal toxicities were monitored by applying multidose regimen (total seven doses, at alternate days) to determine biochemical profiles of serum creatinine and alkaline phosphatase (ALP). The blood was collected by retro-orbital puncture from the mice of different groups after the last administered dosage. The blood was allowed to clot at room temperature, and serum was separated for investigation of creatinine and ALP as per respective guide provided by the manufacturer.

\section{MTT (3-(4,5-dimethylthiazol-2-yl)-2,5- diphenyltetrazolium bromide) assay} Epidermoid carcinoma cell line A253 (ATCC HTB-41 ${ }^{\mathrm{TM}}$ ) was purchased from ATCC (Manassas, VA) and maintained in Roswell Park Memorial Institute (RPMI) 1640 culture medium supplemented with $10 \%$ heat-inactivated fetal calf serum. To perform MTT assay, $5 \times 10^{4}$ cells were transferred to each well of a 96-well plate. The plate was incubated for 24 hours, and then increasing concentrations $(0-60 \mu \mathrm{g} / \mathrm{mL})$ of $\mathrm{POH}$ were added to each well, three wells received medium only with no $\mathrm{POH}$ and served as control. The plate was incubated for 72 hours, and cell proliferation was measured by adding $20 \mu \mathrm{L}$ MTT dye ( $5 \mathrm{mg} / \mathrm{mL}$ in PBS) per well. After further incubation for another 4 hours at $37^{\circ} \mathrm{C}$ in a humidified chamber with $5 \%$ $\mathrm{CO}_{2}$, the formazan crystals formed due to reduction of dye by viable cells in each well were dissolved in $150 \mu \mathrm{L}$ DMSO, and optical density (OD) was read at $620 \mathrm{~nm}$ in a SpectraMax M2 plate reader (Molecular Devices, Sunnyvale, CA).

\section{Effect of POH-PLGA-microparticles in induction of apoptosis in the human epidermoid cancer cell line}

To examine the in vitro inhibitory effect of POH-bearing PLGA microparticles, the microformulation as well as free form drug was incubated with A253 cells $\left(1 \times 10^{8}\right.$ cells $)$ for 12 and 24 hours. After incubation, the cells were scraped, centrifuged, and washed with RPMI medium and lysed with TNN lysis buffer. The lysate was subjected to $12 \%$ sodium dodecyl sulfate polyacrylamide gel electrophoresis, and gels were electroblotted on polyvinylidine fluoride (PVDF) membrane following published protocol. ${ }^{16}$

\section{Animals}

Female Swiss albino mice of weight $20 \pm 2 \mathrm{~g}$ were obtained from the institute's animal house facility. The animals were housed in poly propylene cages on wood powder bedding in an air-conditioned ambience. Animals were quarantined 
on equal light/dark cycles (12/12 hour) and were kept on a pellet diet (Ashirwad, Chandigarh, India) and water ad libitum. Animals were examined for their mortality and morbidity prior to commencement of the study, and only healthy animals were included in the experiments. The techniques used for administration of various formulations as well as sacrifice of the animals were strictly performed following mandates approved by the animal ethics committee (Committee for the Purpose of Control and Supervision of Experiments on Animals, Government of India).

\section{Treatments}

Animals in the resting phase of hair cycle were used in the study. The interscapular regions (over an area of $2 \mathrm{~cm}^{2}$ ) of the experimental animals were shaven using non lubricated electric clippers. The mouse skin tumors were induced by using DMBA as carcinogen following the procedure standardized in our laboratory. ${ }^{10}$ The skin of the shaven dorsal portion of the mice was exposed to DMBA ( $52 \mu \mathrm{g}$ in $200 \mu \mathrm{L}$ acetone) that was applied topically three times a week for 12 weeks. Animals were routinely examined for gross morphological changes on skin and development of tumors. It has been reported that $\mathrm{POH}$ at $10 \mathrm{mM}$ concentration does not cause toxicity in a mice model. ${ }^{17}$ This fact was kept in mind when selecting specific dosage regimens of various cream-based $\mathrm{POH}$ formulations. The formulations of $\mathrm{POH}$ were applied daily with cream base for a period of 7 days after induction of papilloma, with a dose of $10 \mathrm{mM}$ POH in $500 \mu \mathrm{L}$ cream base per animal. Animals were observed for 1 month to examine the effect of various formulations. The animals were divided into the following groups; each consisting of 15 animals: Group I, untreated control (DMBA applied, followed by treatment with cream base only); Group II, sham PLGA microparticles; Group III, control (healthy animals); Group IV, POH (free form); Group V, POH-bearing PLGA microparticles.

\section{Tumor measurement}

Tumor measurement was carried out after 1 month of last dosage application of various $\mathrm{POH}$-based formulations. The diameters of the tumors were measured using a Vernier Caliper, and the tumor volume was determined using the following formula:

$$
\mathrm{V}=\mathrm{D} \times \mathrm{d}^{2} \times \pi / 6
$$

where $\mathrm{V}=$ tumor volume, $\mathrm{D}=$ biggest dimension, and $\mathrm{d}=$ smallest dimension.

\section{Histopathological studies}

Animals were sacrificed and their excised papillomas were immersion fixed in 10\% formalin. Next, the tissue blocks of $3 \times 6 \times 5 \mathrm{~mm}^{3}$ dimensions were processed for paraffin embedding. Thick sections (10 micrometer) were cut with rotary microtome and stained with $\mathrm{H}$ and $\mathrm{E}$ stain. Observations were made under light microscope (Olympus-BX 40-Japan), representative photomicrographs with final magnification of X100 were used for comparative study.

\section{Preparation of nuclear fraction}

The skin/tumor tissues were removed from experimental mice using sharp scalpel blades. The tissue samples were placed on ice, and fat was scraped off before further processing. The samples were homogenized in the presence of protease inhibitor cocktail, and the nuclear fraction was prepared according to the method published elsewhere. ${ }^{18}$

\section{Western blotting}

The tissue homogenate was analyzed for the presence of various apoptotic factors by Western blotting. ${ }^{16}$ Briefly, the protein content of the homogenate was determined by Lowry et al method using bovine serum albumin as a standard. ${ }^{19}$ The homogenate ( $30 \mu \mathrm{g} /$ well) was subjected to PAGE under nondenaturing conditions. The gels were electroblotted onto PVDF membranes, blocked overnight with 5\% non-fat dry milk and probed with appropriate antibodies at the dilutions recommended by the suppliers. To quantify equal loading, membranes were re-probed with $\alpha$-tubulin antibody to determine housekeeping protein tubulin.

\section{Statistical analysis}

One-way analysis of variance was used for comparing the mean values of tumor volume between various groups after ascertaining the homogeneity of variance between treatments. Post-hoc analysis for comparing the two groups was done using the least statistical difference technique. The KaplanMeier analysis was used to determine survival of tumor-free animals, and differences among various experimental groups were analyzed by log-rank test.

\section{Results}

\section{Entrapment efficiency, size, and $\zeta$-potential of PLGA microparticles}

Various PLGA-based microparticle formulations have been widely used for the delivery of antibiotics as well as anticancer drugs to accomplish sustained release of the entrapped 
drugs. ${ }^{14,20}$ The in-house developed PLGA microparticles had entrapment efficiency of $42.4 \pm 3.5$, and size was $768 \pm 215 \mathrm{~nm}$, as revealed by SEM and Nanophox (Sympatec GmbH, Clausthal-Zellerfeld, Germany) size analyzer (Figure 1A and B). As $\zeta$-potential acquired by a small sized particle regulates its half-life in vitro as well as in vivo, it was considered important to determine $\zeta$-potential of the in-house prepared microparticles. The POH-PLGA microparticles used in the present study had a $\zeta$-potential of $-7.6 \pm 0.8 \mathrm{mV}$.

\section{In vitro release kinetics of $\mathrm{POH}$-loaded PLGA microparticles}

In vitro release kinetics of POH-loaded PLGA microparticles was studied at $37^{\circ} \mathrm{C}$ in PBS, pH 7.4. POH-PLGA microparticles showed sustained release, with only $12 \%$ of the entrapped drug leaking out in the initial 18 hours. In the next 12 hours, around $17 \%$ of the total drug was released.
PLGA microparticles showed an initial burst release pattern followed by sustained release kinetics for extended time period. In the present study, PLGA microparticles were found releasing $22 \%$ in the initial 72 hours with an overall total $30 \%$ release in 168 hours (Figure $1 \mathrm{C}$ ).

\section{In vitro and in vivo toxicity of $\mathrm{POH}$-based PLGA microparticle formulation}

Before determining the efficacy of any formulation, it is desirable to assess its intrinsic toxicity. With this aim, POHbearing PLGA microparticle (in-house prepared) toxicity was evaluated, and it was found that POH-bearing PLGA microparticles had induced relatively less percent RBC lysis than free form of the drug (Figure 2). Sham PLGA also induced very negligible lysis.

In another set of experiments, animals treated with multiple dosages of POH-bearing PLGA microparticles were
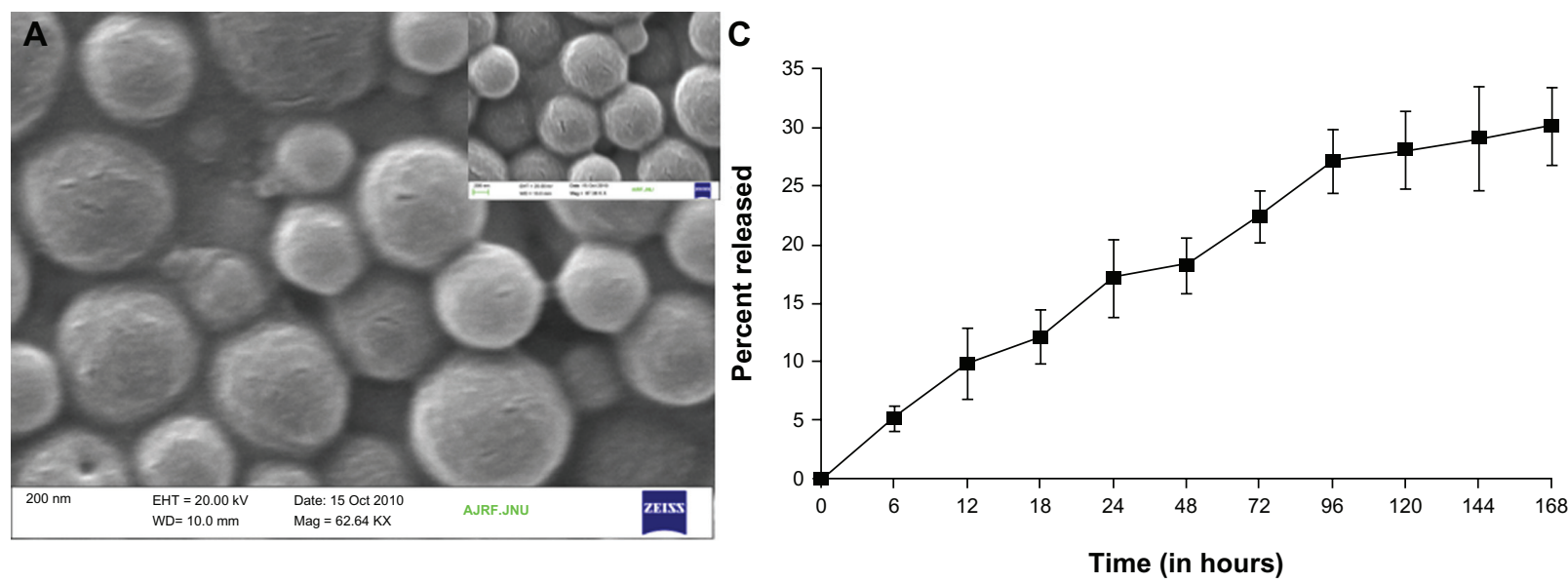

B

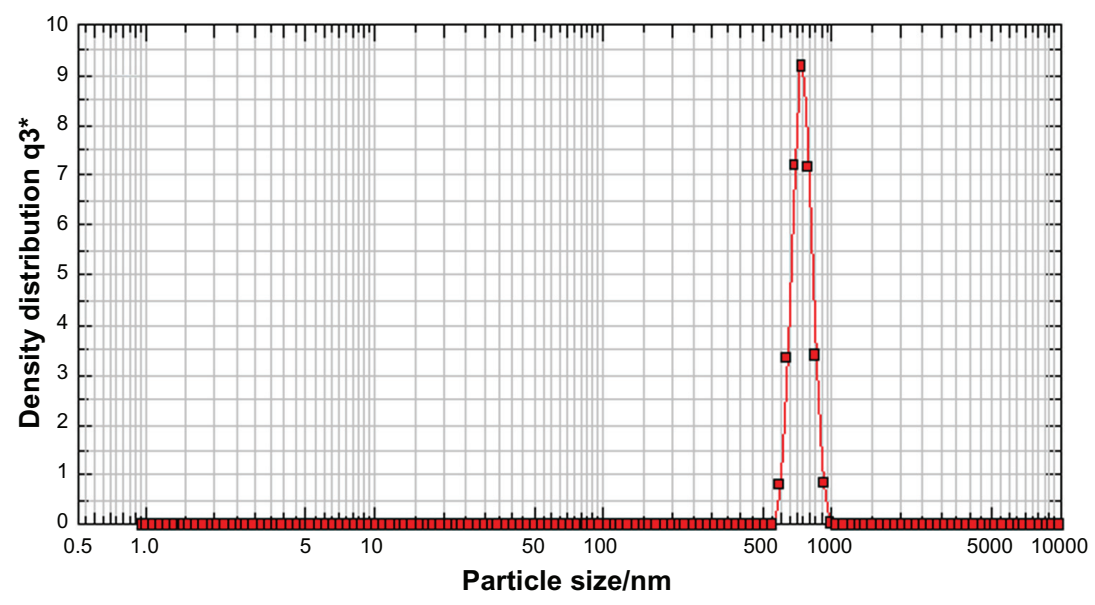

Figure I (A) Scanning electron microscopy image of POH-loaded PLGA microparticles. (B) Corresponding particle analyzer data as obtained by Nanophox (Sympatec $\mathrm{GmbH}$, Clausthal-Zellerfeld, Germany) particle size analyzer. (C) In vitro release kinetics from PLGA microparticles. Release kinetics of POH-PLGA microparticle formulation demonstrates $\sim 30 \%$ release of $\mathrm{POH}$ from the copolymer at 168 hours, when formulation was dispersed in $20 \mathrm{mM}$ phosphate buffer, $\mathrm{pH} 7.4$ at $37^{\circ} \mathrm{C}$.

Note: Data are means \pm standard deviations of three independent experimental values.

Abbreviations: PLGA, poly-lactic glycolic acid; POH, perillyl alcohol; EHT, extra high tension; WD, working distance. 


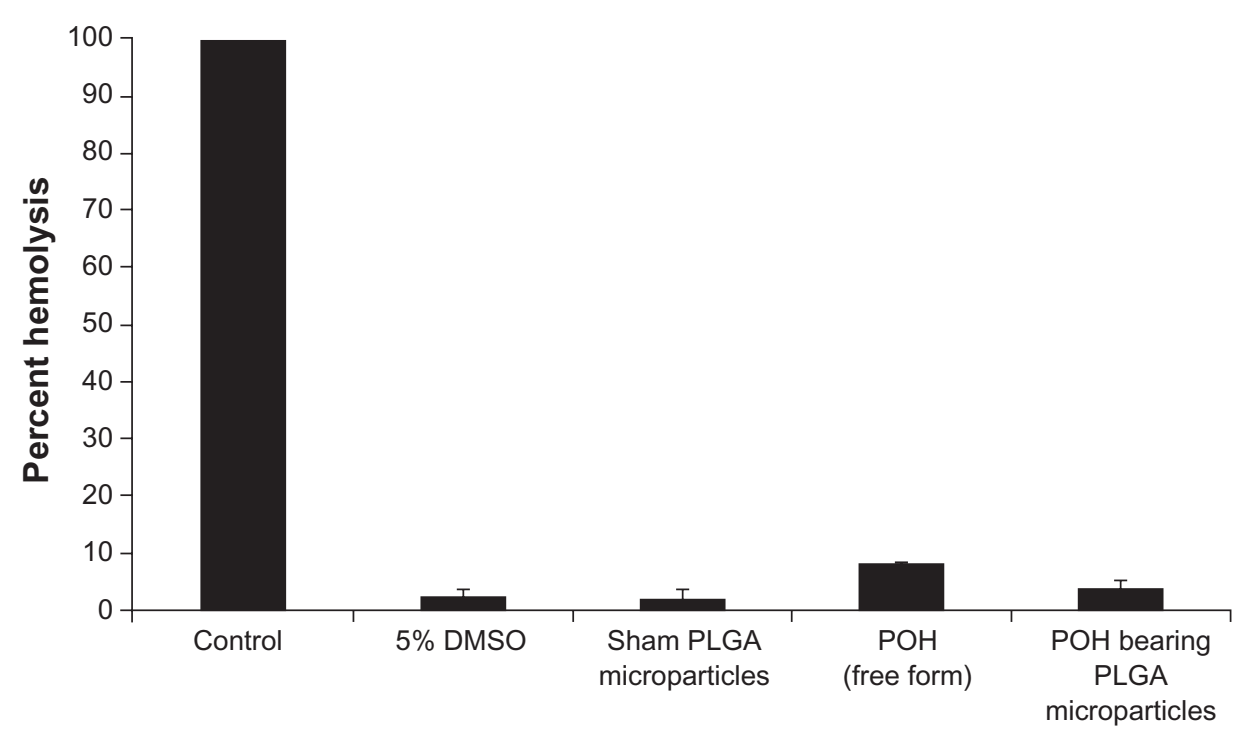

Figure 2 Erythrocyte lysis test: In vitro toxicity was measured by erythrocyte lysis caused by different POH formulations.

Notes: Hemolysis test was performed as described in the Materials and methods section. Data represented here are means of three different experiments \pm standard deviations.

Abbreviations: DMSO, dimethyl sulfoxide; PLGA, poly-lactic glycolic acid; POH, perillyl alcohol.

analyzed for liver as well as renal function test parameters to evaluate in vivo toxicity. As shown in Table 1, POH-bearing PLGA microparticles had comparatively low levels of ALP and creatinine than the free form of the drug. The results established that POH-bearing PLGA microparticles did not have any in vitro and in vivo toxicity and are safe to use.

\section{Cytotoxic effect of POH-bearing PLGA microparticles}

The cytotoxic effect of $\mathrm{POH}$ formulations against epidermoid cancer cells was studied using MTT assay as shown in Figure 3. The data shows that the cytotoxic effect of $\mathrm{POH}$ was significant on epidermoid cancer cells at a concentration of $25 \mu \mathrm{g} / \mathrm{mL}$ and resulted in killing of about $50 \%$ of the cell population.

Nanoparticles are endocytosed by cells faster, ${ }^{20}$ enabling high payload of drug molecules, and exhibit more cytotoxic effect. With this hypothesis, the differential cytotoxicity of free and PLGA microparticle-encapsulated $\mathrm{POH}$ at its halfmaximal inhibitory concentration $\left(\mathrm{IC}_{50}\right)$ was investigated. As shown in Figure 4, free POH caused 50\% cell death, whereas it increased to $\sim 65 \%$ in the POH-PLGA microparticle-treated group in 48 hours incubation (POH-PLGA-microparticle versus free $\mathrm{POH} ; P<0.001)$. In addition, the time-dependent efficacy of $\mathrm{POH}$-bearing microparticles on epidermoid cancer cell system was examined. As shown in Figure 5A, POH-PLGA microparticles enhanced the expression of $\mathrm{p} 21 /$ wafl and bax at 12 hours post-incubation, whereas $\mathrm{POH}$ in free form was not very effective. Similarly, 24 hours post-incubation, the expression of $\mathrm{p} 21 /$ waf1 in cells treated with POH-PLGA microparticles increased further, while the free form drug was not found to be effective (Figure 5B). The results clearly suggest that microparticle encapsulated $\mathrm{POH}$ is delivered efficiently to the cancer cells and can easily modulate various apoptotic factors and eventually results in apoptosis of the cancer cells.

\section{Effect of POH-PLGA microparticles on regression of tumors and survival of animals}

After establishing anticancer efficacy of inhouse-prepared microparticles against cancer cells in vitro, the present study was extended in vivo using a mouse model of skin carcinoma. For this purpose, regression in the volume of DMBA-induced tumors was measured after treatment with various $\mathrm{POH}$ formulations. As shown in Figure 6, the percent regression was much higher in the POH-PLGA microparticle-treated group as compared with those receiving the free form of the

Table I Concentrations of creatinine and ALP in plasma of animals treated with POH-bearing PLGA microparticle formulation

\begin{tabular}{lll}
\hline Groups & Creatinine (mg \%) & ALP (IU/L) \\
\hline Control & $0.32 \pm 0.052$ & $32.46 \pm 2.12$ \\
Sham PLGA microparticles & $0.28 \pm 0.034$ & $36.88 \pm 3.41$ \\
POH (free form) & $0.35 \pm 0.038$ & $44.28 \pm 2.32$ \\
POH-PLGA microparticles & $0.31 \pm 0.011$ & $34.25 \pm 2.14$ \\
\hline
\end{tabular}

Abbreviations: ALP, alkaline phosphatase; PLGA, poly-lactic glycolic acid; POH, perillyl alcohol. 


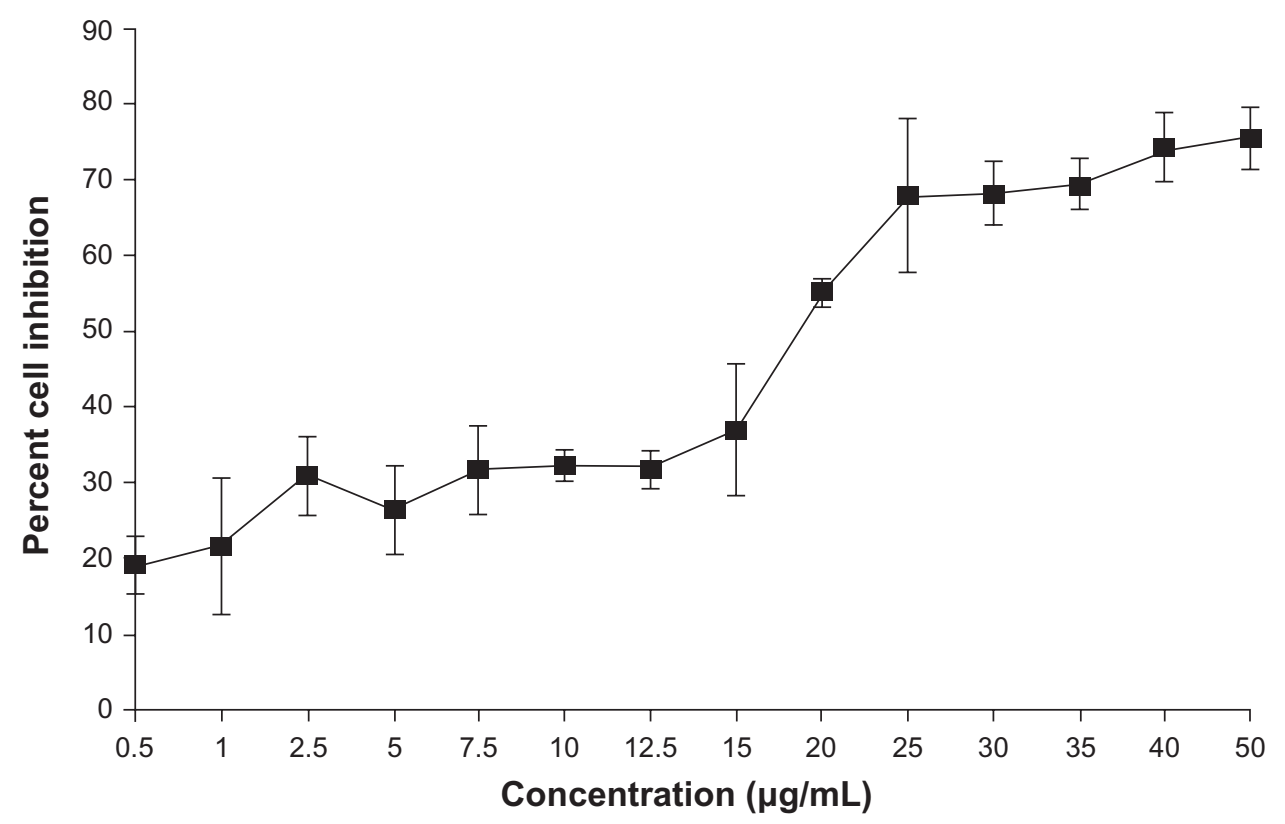

Figure 3 In vitro cytotoxicity of perillyl alcohol against A253 cell line as revealed by MTT assay.

Notes: MTT assay was performed as described in the Materials and methods section. Data represented here are means of three different experiments \pm standard deviations.

Abbreviation: MTT, 3-(4,5-dimethylthiazol-2-yl)-2,5-diphenyltetrazolium bromide.

drug $(P$ value $<0.001$; POH-PLGA-microparticle versus free $\mathrm{POH})$. The treatment with microparticle-based $\mathrm{POH}$ formulation resulted in $80 \%$ tumor regression, while the free form of drug was able to regress tumor by $65 \%$ only. The sham microparticles were devoid of any significant anticancer activity.

Next, the efficacy of POH-PLGA microparticles was assessed in terms of the survival of treated animals. Survival graph shows the augmentation of anticancer efficacy of PLGA-encapsulated POH microparticles as well as free form

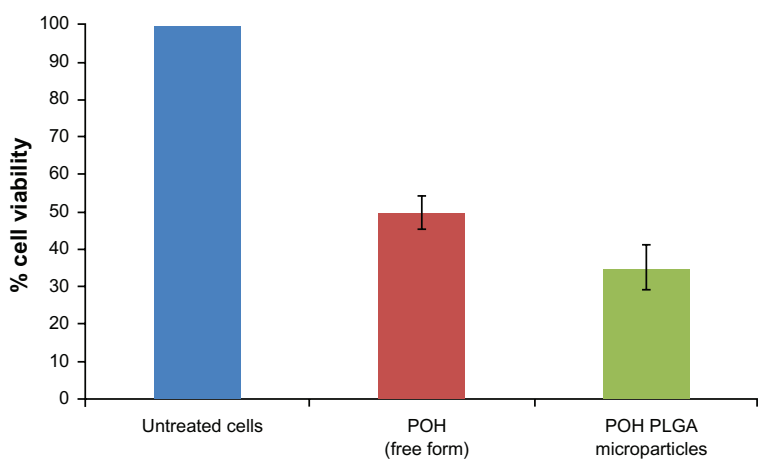

Figure 4 Determination of differential cytotoxicity of various formulations of $\mathrm{POH}$ using MTT assay. Cells were incubated with various formulations of $\mathrm{POH}$ for 48 hours.

Notes: The percentage cell viability was measured with MTT assay as described in the Materials and methods section. Data represented here are means of three different experiments \pm standard deviations ( $\mathrm{POH}-\mathrm{PLGA}$ microparticle versus free form $\mathrm{POH} P<0.00 \mathrm{I})$.

Abbreviations: MTT, 3-(4,5-dimethylthiazol-2-yl)-2,5-diphenyltetrazolium bromide; PLGA, poly-lactic glycolic acid; POH, perillyl alcohol.
POH against DMBA-induced tumorogenesis at different time points. POH-encapsulated PLGA microparticles showed $80 \%$ survival, whereas the group receiving free form of $\mathrm{POH}$ resulted in only $40 \%$ survival in 13 weeks (POH-PLGA microparticles versus free POH; $P<0.05$ ). None of the animals survived beyond 12 weeks in the control group that was treated with cream base only $(P<0.01)$ (Figure 7$)$.

\section{Histopathological analysis}

For histopathological studies, skin tissue samples were isolated from animals of various groups and analyzed following the protocol described in Materials and methods. Histopathological studies of tumors identified papillomas in various groups. However, the relative frequency of the individual tumor types differed among animals receiving different treatments. In the skin of healthy animals, there were only a few cell layers thick keratinocytes with mild keratin and pilosebaceous units (Figure 8A), whereas mice treated with DMBA followed by no $\mathrm{POH}$ treatment (positive control) had obvious profuse papillomatous growth with complex fibrovascular core, prominent acanthosis and keratin perl (Figure 8B). On the other hand, in the free $\mathrm{POH}$ treated group, a large amount of keratin and marked acanthosis was visible as depicted in Figure 8C, while hyperkeratosis, only mild acanthosis, and thin but long papillary growth was observed in animals treated with POH-PLGA microparticles (Figure 8D). 


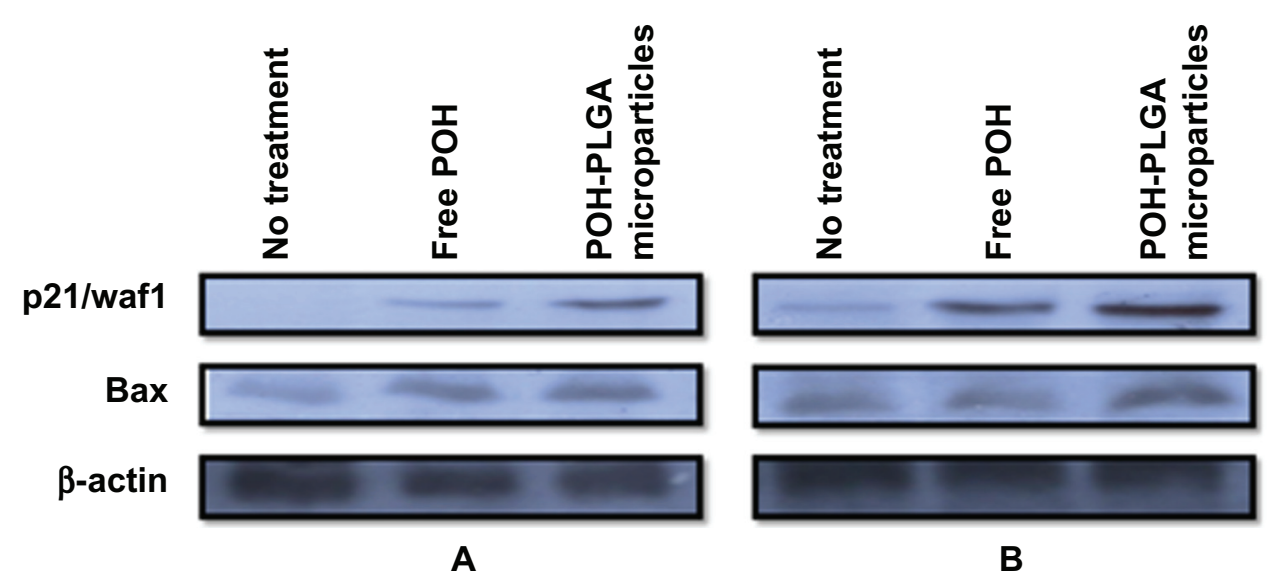

Figure 5 Effect of $\mathrm{POH}$-bearing formulations on expression of pro-apoptotic molecules. A253 cancer cells were treated with $\mathrm{POH}$-bearing microformulations for different time periods, and cell lysates were used to examine the expression of apoptotic molecules. (A) Expression profile of apoptotic factors at 12 hours post-incubation. (B) Expression profile of apoptotic factors at 24 hours post-incubation.

Abbreviations: PLGA, poly-lactic glycolic acid; $\mathrm{POH}$, perillyl alcohol.

\section{Western blot analysis}

DMBA is generated from incomplete combustion of various organic substances. It is cytotoxic, mutagenic, and inflicts carcinogenic effects in humans and animal models..$^{21,22}$ DMBA induces skin papillomas in animal models by upregulating p53 mutant in cancer cells. As shown in Figure 9, POHPLGA microparticles downregulate p53-mutant expression significantly when compared with free form of $\mathrm{POH}$. Western blot analysis was also performed to assess expression of p53wt and p21/waf1. POH-PLGA microparticles showed

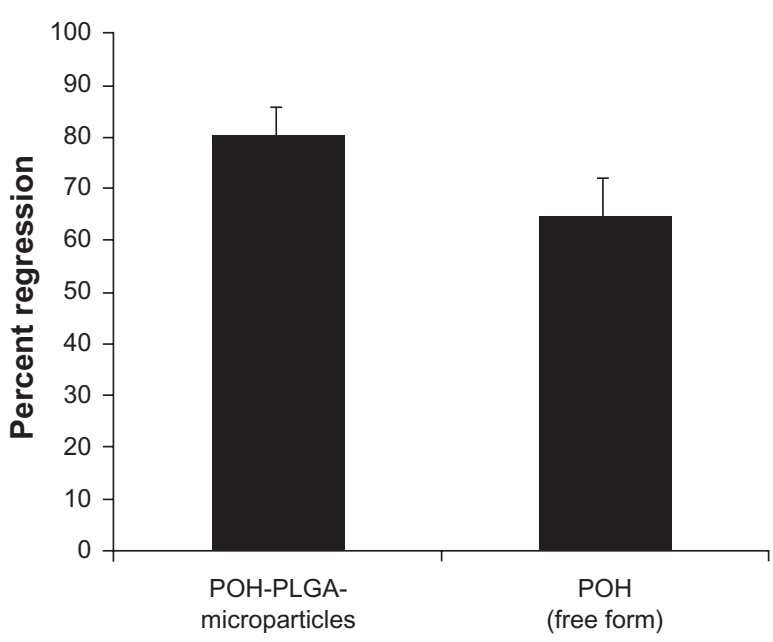

Figure 6 Chemotherapeutic effect of two different formulations of $\mathrm{POH}$ in regression of tumors in treated animals.

Notes: Percentage regression was calculated to analyze the most effective formulation of $\mathrm{POH}$. It was assessed by measuring the size with Vernier calipers, and tumor volume was calculated as given in the Materials and methods section (POHPLGA microparticles versus free form POH; $P<0.001$ ). Sham-PLGA microparticles behaved as control.

Abbreviations: PLGA, poly-lactic glycolic acid; POH, perillyl alcohol. significant upregulation of $\mathrm{p} 53 \mathrm{wt}$ and $\mathrm{p} 21 / \mathrm{waf} 1$ expression in comparison with free form POH (POH-PLGA microparticles versus free POH; $P<0.05$ ).

\section{Discussion}

Cell-cycle progression in eukaryotes is regulated by several key factors that determine whether the cell will re-enter the cycle, withdraw from it, or undergo differentiation. A slight deviation from the normal course may result in uncontrolled proliferation of the cells that eventually form the basis of cell transformation. In general, malignant state is preceded by several discernible stages, including initiation of DNA damage followed by tumor promotion both in vivo and cancer cells growing in vitro.

A large number of phytochemicals have been reported to prevent or arrest uncontrolled growth of cells through a variety of operative mechanisms. Current global focus is on natural products as a means to control various types of cancers. Some plant-based products possess strong antioxidant properties and have the potential to control or even reverse the process of carcinogenesis and emerge as potential alternatives to chemotherapeutic anticancer agents. ${ }^{23}$ However, before developing such potential phytochemicals as prospective therapeutic agents, certain challenges such as palatability, poor solubility, and other related problems have to be addressed. Some of these can be generally circumvented through the use of an appropriate delivery system that has potential to modify the pharmacokinetics of the drug and facilitate its controlled and even preferential release at the desired tumor site. 


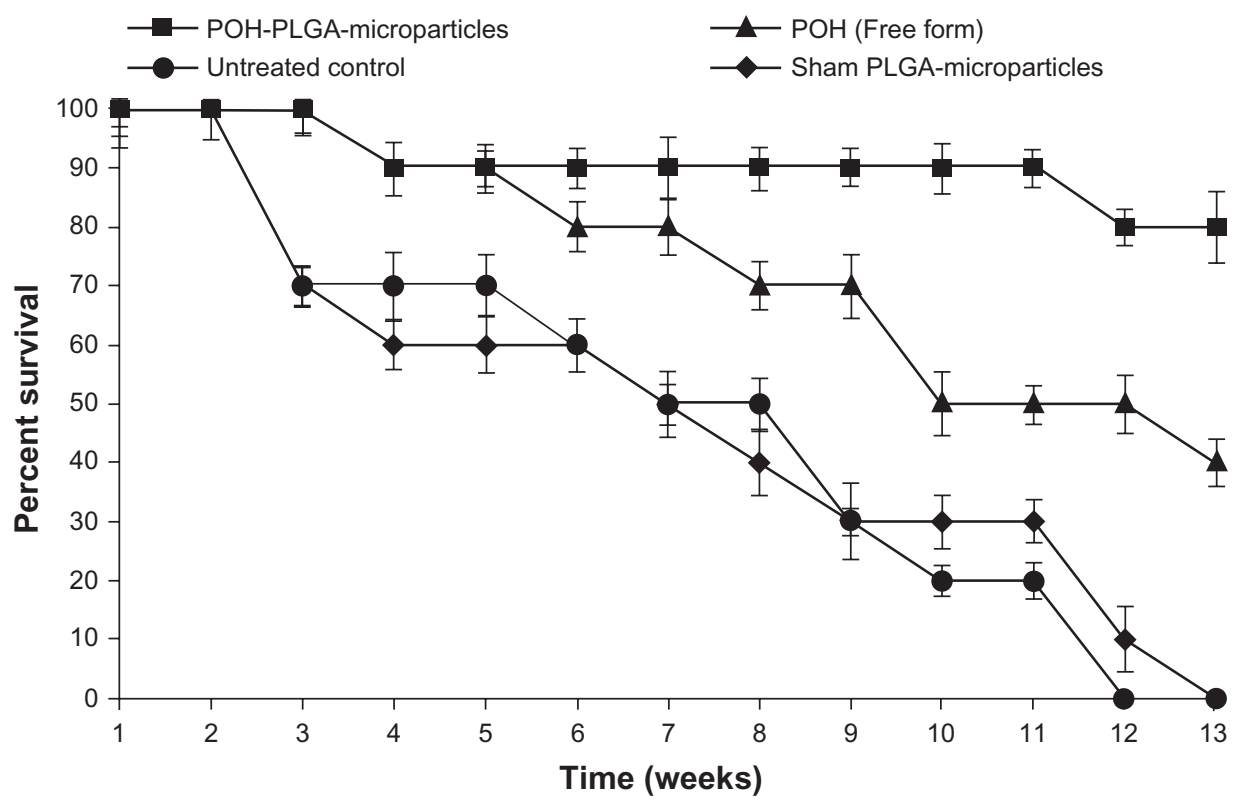

Figure 7 Effect of POH-bearing PLGA formulations on survival of DMBA-induced skin papilloma-carrying animals.

Notes: Kaplan-Meier graph shows the efficacy of various $\mathrm{POH}$ formulations in terms of percentage survival after treatment at different time intervals. The study was continued for a period of 13 weeks as described in the Materials and methods section (POH-PLGA microparticles versus free form POH; $P<0.05$ ). Abbreviations: PLGA, poly-lactic glycolic acid; $\mathrm{POH}$, perillyl alcohol.

$\mathrm{POH}$, a dietary constituent and a mono-terpene isolated from cherries, had been used widely as an anticancer agent against various types of cancer in animal models. ${ }^{4,24,25} \mathrm{POH}$ is known to arrest cells in $\mathrm{G} 0 / \mathrm{G} 1$ phase and induce apoptosis. ${ }^{26}$ Although $\mathrm{POH}$ is a very effective chemotherapeutic agent, its anticancer properties have not been explored completely. In fact, certain intrinsic properties of $\mathrm{POH}$ such as small size, poor solubility in aqueous solution, and bioavailability restrict attainment of its effective concentration at the targeted site and thus limit its application in cancer treatment. Earlier studies suggest that localized delivery at the targeted site could be enhanced significantly by the use of microparticle-based drug delivery and eventually can be successfully employed in tumor therapy. ${ }^{27,28}$ It has recently been demonstrated that naturally occurring phytochemicals can be encapsulated, covalently attached, and adsorbed onto microparticles in order to surmount drug-solubility problems. ${ }^{10,29,30}$ The constituents of the microparticle-based delivery system can also facilitate co-solubilization of the compound/drug in question. This approach has significant implication in cancer therapeutics because more than nearly half of the phytochemicals showing anticancer activity have solubility constraints. ${ }^{31}$ Nanosize range of the microparticles entails a high surface area that not only provides sustained drug release but also provides a useful strategy for their functionalization. The present study focuses on development and characterization of various $\mathrm{POH}$-bearing microparticle formulations and their evaluation in chemotherapy against DMBA-induced skin papilloma in Swiss albino mice.

The POH-bearing PLGA microparticle formulation was characterized for sizing using electron microscopy, and its surface charge properties were assessed on the basis of $\zeta$-potential. The biophysical properties of the novel formulation were also assessed. Further, in vitro anticancer efficacy of PLGA based POH formulation was examined against human epidermoid cancer cell line (A253). Finally, the in vivo therapeutic potential of POH-PLGA microparticle formulations was evaluated on the basis of tumor size regression, survival, histology, and expression profiles of various apoptotic molecules.

To optimize the size and loading efficiency of the active components, a series of PLGA-based microparticles with varying compositions were prepared. The size of the inhouse prepared microparticles was standardized by incorporating PLGA of various molecular- masses. Further, the entrapment efficiency at various added weight percentage values of POH to PLGA was examined. It was found that $50 \%-70 \%$ weight of $\mathrm{POH}$ to that of PLGA leads to entrapment efficiency of around $42.4 \pm 3.5$ with the size of prepared microparticles in the range of $768 \pm 215 \mathrm{~nm}$ (Figure 1A and B). This formulation of POH-PLGA was used in subsequent studies. 


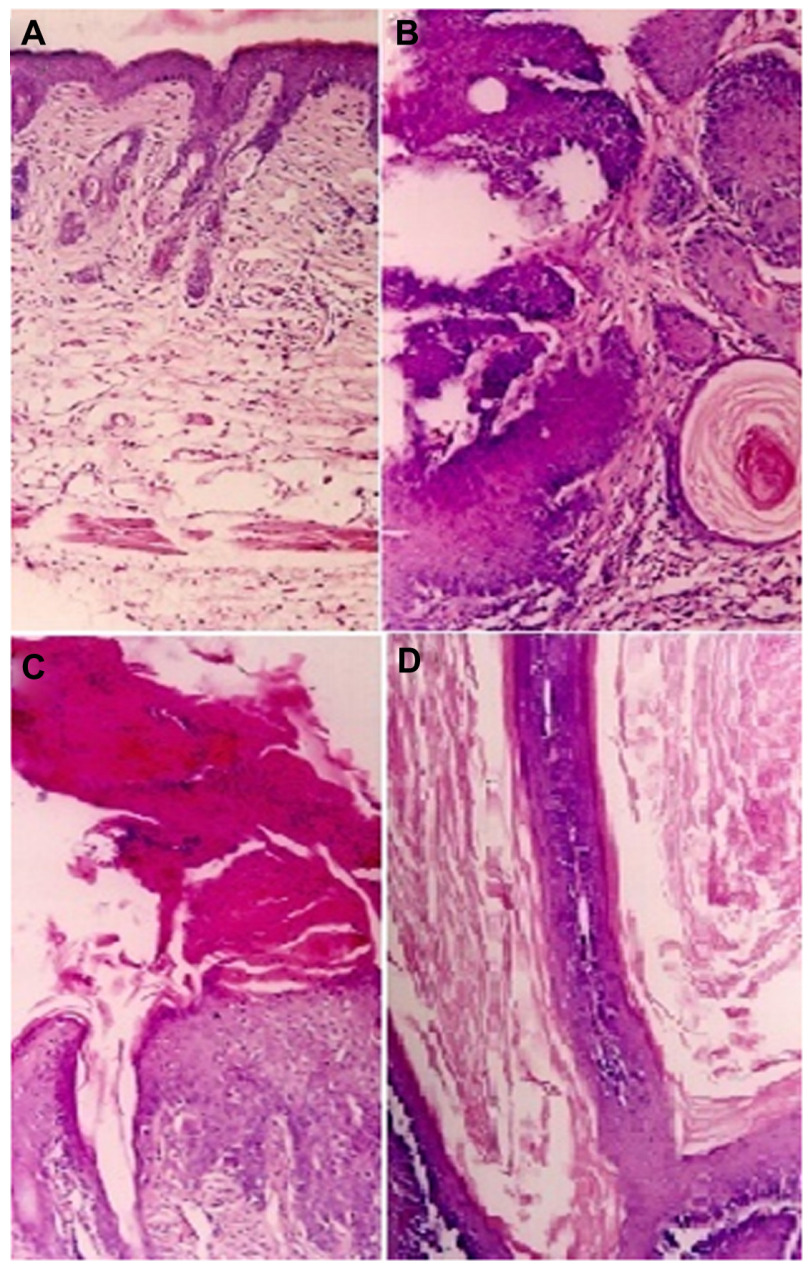

Figure 8 Photomicrographs of hematoxylin/eosin-stained sections, showing: (A) normal smooth skin of healthy animals with keratinocytes of only a few cell layers thick, mild keratin, and pilosebaceous units; (B) the positive control (DMBA treated animals followed by no $\mathrm{POH}$ treatment) has profuse papillomatous growth with complex fibrovascular core, prominent acanthosis, and keratin perl; (C) treatment with free form of $\mathrm{POH}$ resulted in large amount of keratin, and marked acanthosis; while (D) the treatment with PLGA microsphere encapsulated $\mathrm{POH}$ resulted in hyperkeratosis, mild acanthosis, and thin but long papillary growth.

Note: Magnification 100x.

Usage of the free form drug does not allow attainment of effective concentration unless a large payload is administered, which ironically results in many untoward effects. In such cases, drug-delivery systems offer help by providing continuous and constant supply of the therapeutics for an extended time period. The hydrophobic POH is likely to be physically dispersed by its encapsulation throughout the matrix of PLGA particle. The release kinetics of the entrapped drug showed an overall slow and sustained release that was likely to be regulated by rate of polymer biodegradation. The release kinetics of entrapped drug was determined by incubating the drug-bearing microparticles in PBS, $\mathrm{pH} 7.4$ at $37^{\circ} \mathrm{C}$ to simulate physiological conditions. The amount of released $\mathrm{POH}$ was estimated by reverse phase HPLC analysis.
A linear release of $\mathrm{POH}$ accounted for around $22 \%$ of the total POH entrapped in PLGA microparticles. In concurrence with earlier reports, where PLGA with the 50:50 ratio of lactic:glycolic acid composition was found to degrade in 1-2 months, ${ }^{32}$ the in-house-prepared PLGA microparticles acquired steady release and showed $30 \%$ release of entrapped molecules in a time span of 7 days (Figure 1C).

Both in vitro and in vivo toxicity data revealed that $\mathrm{POH}-$ bearing PLGA microparticles had negligible toxicity when compared with free form of the drug and thus assures its safety (Figure 2 and Table 1). The cytotoxicity of free $\mathrm{POH}$ was next examined, and it was found that $\mathrm{POH}$ had an $\mathrm{IC}_{50}$ of $25 \mu \mathrm{g} / \mathrm{mL}$ against A253 human epidermoid cancer cells (Figure 3). This concentration was used to analyze differential cytotoxicity of free and PLGA microformulation-encapsulated $\mathrm{POH}$ and their comparative potential in induction of apoptosis in epidermoid cancer cell line. Due to faster uptake and subsequent release of the drug by POH-PLGA microparticles, it exerts more cytotoxic effect on epidermoid cancer cells than its free form (Figure 4). This observation could be explained on the premise that application of POH-PLGA microparticles delivers a high amount of active drug at the tumor site mainly. Further, polymer conjugation of low molecular weight drugs alters their biodistribution; enabling their passive targeting and reducing access to sites of toxicity. ${ }^{33}$ Microparticle-based drug formulations entertain a special status in improving the efficacy of drug against many types of cancers. Therefore, the present study was extended to gain insight in POH-PLGA microparticle-mediated modulation of various proteins involved in the apoptosis of A253 cells. Western blot analysis was performed to determine levels of various cell cycle and apoptosis regulating factors. As evident from Figure 5A, incubation of $\mathrm{POH}$-microparticle formulation with epidermoid cancer cells resulted in enhanced expression of p21/waf1 when compared with free form of $\mathrm{POH}$. The expression of another important pro-apoptotic factor Bax was also analyzed in A253 cells upon their incubation with $\mathrm{POH}$-microparticle formulations. The POH-microparticle formulation up-regulates the expression of Bax, thereby enhancing apoptosis induction in cancer cells (Figure 5A). In the control groups (cells treated with sham PLGA microparticles), the expression profile of these molecules was not significantly affected when compared with POH-PLGA microparticles, suggesting that microparticle-based formulation is effective in upregulating p21/waf1 and Bax gene expression. The effect of POH-PLGA microparticles on expression of pro-apoptotic molecules was more prominent after 24 hours incubation (Figure 5B). Interestingly, the POH-PLGA microparticle was 

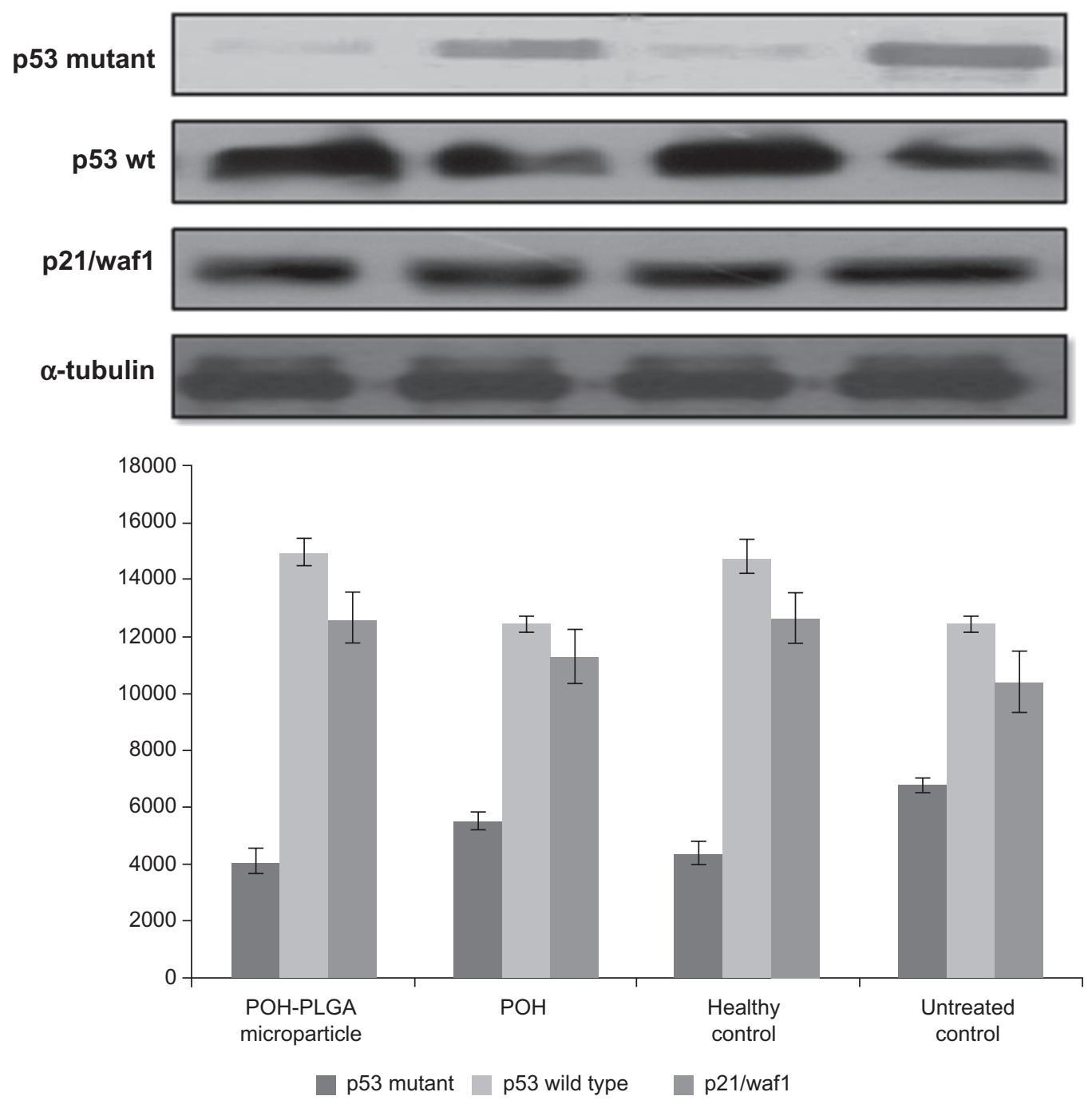

Figure 9 Effect of different POH-bearing formulations on the expression of different apoptosis-regulating molecules in treated animals.

Notes: Skin papilloma cell lysates were prepared as described in the Materials and methods section. Cell lysate was resolved and analyzed using SDS-PAGE. To ensure equal loading, membranes were re-probed for the presence of tubulin, a housekeeping protein, using $\alpha$-tubulin antibody.

Abbreviations: PLGA, poly-lactic glycolic acid; POH, perillyl alcohol.

equally successful in downregulating p53-mutant expression in A253 cancer cells (data not shown). Of note, both p53 and/or p21-dependent and -independent pathways have been previously reported to be involved in $\mathrm{POH}$-induced cell cycle arrest and apoptosis in cancer cells; however, the extent of the cell responses varied and can be attributed to the mechanism operative in a specific cell type involved and the doses of the drug used in the study. ${ }^{34,35}$

DMBA, a polycyclic aromatic hydrocarbon, is metabolized to chemically reactive electrophile and initiate a cascade of reaction that eventually results in carcinogenesis by covalent interaction(s) with DNA. ${ }^{22}$ Further investigations suggest that it induces skin papilloma in animal models by mutagenesis in Ha-ras oncogene. ${ }^{36}$ Upon topical application of POH-PLGA microparticles, $80.8 \pm 5.2$ regression in tumor size was observed after the treatment, whereas free form POH showed only 65.1 \pm 7.1 regression (Figure 6). Increased regression in the POH-PLGA microparticle group could be attributed to sustained release of $\mathrm{POH}$. Interestingly, the tumor regression results were found to have great correlation with survival rate of the treated animals. In case of POHPLGA microparticles, survival rate was highest ( $80 \%)$ over a period of 13 weeks when compared with survival rate of the animals treated with free form POH (40\%) (Figure 7). Further, histopathological analysis of the skin tissues isolated from treated animals was performed and the results clearly demonstrated that free form $\mathrm{POH}$ had more acanthosis and papillary growth than POH-bearing PLGA microformulations (Figure 8). The pro-apoptotic gene p53 regulates the balance between cell proliferation and cell death. ${ }^{37,38}$ It has 
been observed that the p53 gene gets mutated in most types of the malignancies, including sarcomas. Upon exposure to a potent carcinogen, the toxic insult of the cell is generally nullified by a chain of cell cycle regulatory events programmed to check the uncontrolled proliferation by a suitable repair mechanism. ${ }^{39}$ The failure to induce expression of functional p53wt leads to disregulation of cell cycle arrest or cell death and tumor progression. The results of the present study showed decreased p53-mutant expression upon treatment with POH-bearing PLGA microparticles. Also, higher efficacy of the microparticle-based formulation of $\mathrm{POH}$ can be correlated with upregulated expression of p53wt and p21/ waf1 genes (Figure 9).

The survival data further establish higher supremacy of POH-PLGA microparticles over its free form. The observed higher efficacy of POH-bearing PLGA microparticle formulations can possibly be attributed to the greater bioavailability of $\mathrm{POH}$ and its accumulation at the tumor site. Finally, it can be inferred that microparticle-based formulations not only overcome the solubility constraints of the poorly water soluble $\mathrm{POH}$ but also facilitate its release in regulated fashion that eventually results in better efficacy of $\mathrm{POH}$ in terms of tumor regression and survival of treated animals.

\section{Conclusion}

Entrapment of POH in PLGA microparticles makes its release more sustained, thereby facilitating accumulation of required concentration at the target site. As revealed by cytotoxic assay, POH-bearing PLGA microparticles showed greater growth inhibition of human epidermoid cancer cell line than free form POH. Further, higher tumor regression, increased survival, histopathological study, and Western blot profiles of various cell cycle regulatory proteins clearly suggest that POH-PLGA microparticles offer better efficacy than free form $\mathrm{POH}$ against DMBA-induced skin papillomas in animal models.

\section{Acknowledgments}

The authors are thankful to their coordinator, Professor M Saleemuddin, for providing the facilities. Mohammad Farazuddin acknowledges financial support in the form of SRF provided from CSIR (India). Financial assistance provided by UGC and DST under the FIST program is greatly acknowledged.

\section{Disclosure}

The authors claim that there is no conflict of interest in this work.

\section{References}

1. Haag JD, Gould MN. Mammary carcinoma regression induced by perillyl alcohol, a hydroxylated analogue of limonene. Cancer Chemother Pharmaco. 1994;34(6):477-483.

2. Stark MJ, Burke YD, McKinzie JH, Ayoubi AS, Crowell PL. Chemotherapy of pancreatic cancer with the monoterpene perillyl alcohol. Cancer Lett. 1995;96(1):15-22.

3. James T, Belanger ND. Perillyl alcohol: applications in oncology. Alternc Med Rev. 1998;3(6):448-457.

4. Kelloff GJ, Boone CW, Crowell JA, et al. New agents for cancer chemoprevention. J Cell Biochem Suppl. 1996;26:1-28.

5. Muller RH, Radtke M, Wissing SA. Solid lipid nanoparticles (SLN) and nanostructured lipid carriers (NLC) in cosmetic and dermatological preparations. Adv Drug Deliv Rev. 2002;54(1):131-155.

6. Spiclin P, Homar M, Zupancic-Valant, Gasperlin M. Sodium ascorbyl phosphate in topical microemulsions. Int Pharm. 2003;256(1-2): $65-73$.

7. Fang J, Hwang T, Huanga Y, Fang C. Enhancement of the transdermal delivery of catechins by liposomes incorporating anionic surfactants and ethanol. Int J Pharm. 2006;310(1-2):131-138.

8. Betz G, Aeppli A, Menshutina N, Leuenberger H. In vivo comparison of various liposome formulations for cosmetic application. Int J Pharm. 2005;296(1-2):44-54.

9. Kirjavainen M, Urtti A, Valjakka-Koskela R, Kiesvaara J, Monkkonen J. Liposome skin interactions and their effects on the skin permeation of drugs. Eur J Pharm Sci. 1999;7(4):279-286.

10. Khan A, Shukla Y, Kalra N, et al. Potential of diallyl sulfide bearing $\mathrm{pH}$-sensitive liposomes in chemoprevention against DMBA-induced skin papilloma. Mol Med. 2007;13(7-8):443-451.

11. Nair LS, Laurencin CT. Biodegradable polymers as biomaterials. Prog Polym Sci. 2007;32(89):762-798.

12. Jeffery H, Davis SS, O'Hagan DT. The preparation and characterization of PLGA nanoparticles: II. The entrapment of model protein using (water in oil) in water emulsion solvent evaporation technique. Pharm Res. 1993;10(3):362-368.

13. Farazuddin M, Alam M, Khan AA, et al. Efficacy of amoxicillin bearing microsphere formulation in treatment of Listeria monocytogenes infection in Swiss albino mice. J Drug Target. 2010;18(1):45-52.

14. Tao L, Pereira MA. Quantification of carvone, cineole, perillaldehyde, perillyl alcohol and sobrerol by isocratic high-performance liquid chromatography. J Chromtogr A. 1998;793(1):71-76.

15. Khan MA, Syed FM, Nasti HT, et al. Use of tuftsin bearing nystatin liposomes against an isolate of Candida albicans showing less in vivo susceptibility to amphotericin B. J Drug Target. 2002;11(2): 93-99.

16. Towbin H, Staehelin T, Gordon J. Electrophoretic transfer of proteins from polyacrylamide gels to nitrocellulose sheets: procedure and some applications. Proc Natl Acad Sci U S A. 1979;76(9):4350-4354.

17. Barthelman M, Chen W, Gensler HL, Huang C, Dong Z, Bowden GT. Inhibitory effects of perillyl alcohol on UVB-induced murine skin cancer and AP-1 transactivation. Cancer Res. 1998;58(4): 711-716.

18. Serpi R, Piispala J, Jarvilehto M, Vahakangas K. Thapsigargin has similar effect on $\mathrm{p} 53$ protein response to benzo[a]pyrene-DNA adducts as TPA in mouse skin. Carcinogenesis. 1999;20(9):1755-1760.

19. Lowry OH, Rosenbrough NK, Farr AL, Randall RJ. Protein measurement with folin phenol reagent. J Biol Chem. 1951;193(1):265-275.

20. Kocbek P, Obermajer N, Cegnar M, Kos J, Kristl J. Targeting cancer cells using PLGA nanoparticles surface modified with monoclonal antibody. J Control Rel. 2007;120(1-2):18-26.

21. Guerin MR. Energy sources of polycyclic aromatic hydrocarbons. In: Gelboin HV, Tso POP, editors. Polycyclic Hydrocarbons in Cancers. New York: Academic Press; 1978:3-42.

22. Dipple A, Mosschel RC, Bigger CAH. Polynuclear aromatic carcinogens. In: Charles E, Searle, editors. Chemical Carcinogens. Washington, DC: American Chemical Society; 1984:41-126. 
23. Reddy L, Odhav B, Bhoola KD. Natural products for cancer prevention: a global perspective. Pharmacol Ther. 2003;99(1):1-13.

24. Broitman SA, Wilkinson J 4th, Cerda S, Branch SK. Effects of monoterpenes and mevinolin on murine colon tumor CT-26 in vitro and its hepatic "metastases" in vivo. Adv Exp Med Biol. 1996;401: $111-130$.

25. Lantry LE, Zhang Z, Gao F, et al. Chemopreventive effect of perillyl alcohol on 4-(methylnitrosamino)-1-(3-pyridyl)-1-butanone induced tumorigenesis in $(\mathrm{C} 3 \mathrm{H} / \mathrm{HeJ} \mathrm{X} \mathrm{A} / \mathrm{J}) \mathrm{F} 1$ mouse lung. $J$ Cell Biochem Suppl. 1997;27:20-25.

26. Clark SS, Perman SM, Sahin MB, Jenkins GJ, Elegbede JA. Antileukemia activity of perillyl alcohol (POH): uncoupling apoptosis from $\mathrm{G} 0 / \mathrm{G} 1$ arrest suggests that the primary effect of $\mathrm{POH}$ on $\mathrm{Bcr} /$ Abl-transformed cells is to induce growth arrest. Leukemia. 2002;16: 213-222.

27. Vicent MJ, Duncan R. Polymer conjugates: nanosized medicines for treating cancer. Trends Biotechnol. 2006;24(1):39-47.

28. Nie S, Xing Y, Kim GJ, Simons JW. Nanotechnology applications in cancer. Annu Rev Biomed Eng. 2007;7(6):833-837.

29. Alam M, Farazuddin M, Owais M. Potential use of liposomal diallyl sulfide in the treatment of experimental murine candidiasis. Biosci Rep. 2010;30(4):223-231

30. Alam M, Dwivedi V, Khan AA, Mohammad O. Efficacy of niosomal formulation of diallyl sulfide against experimental candidiasis in Swiss albino mice. Nanomedicine. 2009;4(7):713-724.
31. Merisko-Liversidge E, Liversidge GG, Cooper ER. Nanosizing: a formulation approach for poorly-water-soluble compounds. Eur $J$ Pharm Sci. 2003;18(2):113-120.

32. Middleton JC, Tipton AJ. Synthetic biodegradable polymers as orthopedic devices. Biomaterials. 2000;21(23):2335-2346.

33. Duncan R. Polymer conjugates as anticancer nanomedicines. Nature Reviews. 2006;6:688-701.

34. Clark SS, Perman SM, Sahin MB, Jenkins GJ, Elegbede JA. Antileukemia activity of perillyl alcohol (POH): uncoupling apoptosis from $\mathrm{G} 0 / \mathrm{G} 1$ arrest suggests that the primary effect of $\mathrm{POH}$ on $\mathrm{Bcr} / \mathrm{Abl}$-transformed cells is to induce growth arrest. Leukemia. 2002;16(2): 213-222.

35. Ariazi EA, Satomi Y, Ellis MJ, et al. Activation of the transforming growth factor beta signaling pathway and induction of cytostasis and apoptosis in mammary carcinomas treated with the anticancer agent perillyl alcohol. Cancer Res. 1999;59(8):1917-1928.

36. Bizub D, Wood AW, Skalka AM. Mutagenesis of the Ha-ras oncogene in mouse skin tumors induced by polycyclic aromatic hydrocarbons. Proc Natl Acad Sci U SA. 1986;83:6048-6052.

37. Croce CM. Oncogenes and cancer. N Engl J Med. 2008;358(5): 502-511.

38. Knudson AG. Two genetic hits (more or less) to cancer. Nat Rev Cancer. 2001;1(2):157-162.

39. Branzei D, Foiani M. Regulation of DNA repair throughout the cell cycle. Nat Rev Mol Cell Biol. 2008;9(4):297-308.
International Journal of Nanomedicine

\section{Publish your work in this journal}

The International Journal of Nanomedicine is an international, peerreviewed journal focusing on the application of nanotechnology in diagnostics, therapeutics, and drug delivery systems throughout the biomedical field. This journal is indexed on PubMed Central,

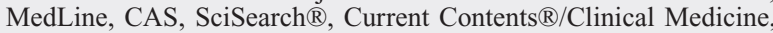

\section{Dovepress}

Journal Citation Reports/Science Edition, EMBase, Scopus and the Elsevier Bibliographic databases. The manuscript management system is completely online and includes a very quick and fair peer-review system, which is all easy to use. Visit http://www.dovepress.com/ testimonials.php to read real quotes from published authors. 